\title{
ON EXPONENTIAL STABILITY OF LINEAR NON-AUTONOMOUS FUNCTIONAL DIFFERENTIAL EQUATIONS OF NEUTRAL TYPE
}

\begin{abstract}
General linear non-autonomous functional differential equations of neutral type are considered. A novel approach to exponential stability of neutral functional differential equations is presented. Consequently, explicit criteria are derived for exponential stability of linear non-autonomous functional differential equations of neutral type. A brief discussion to the obtained results and illustrative examples are given.
\end{abstract}

\section{INTRODUCTION}

Functional differential equations of neutral type have numerous applications in science and engineering. They are used as models of lossless transmission lines, partial element equivalent circuits [2], steam or water pipes, heat exchangers [15] and control of constrained manipulators with delay measurements in mechanical engineering [19].

Problems of stability of functional differential equations of neutral type have been investigated intensively during the past decades, see e.g. [1]-[2], [4]-[10], [12]-[20] and references therein. In this paper, we investigate exponential stability of general linear neutral non-autonomous functional differential equations of the form

$$
\frac{d}{d t} \mathcal{D}\left(t, x_{t}\right)=\mathcal{L}\left(t, x_{t}\right), \quad t \geq \sigma,
$$

where, for each $t \in \mathbb{R}, x_{t}(\cdot) \in \mathcal{C}:=C\left([-h, 0], \mathbb{R}^{n}\right)$ is defined by $x_{t}(\theta):=x(t+\theta), \theta \in[-h, 0]$ with given $h>0, \mathcal{D}(\cdot, \cdot): \mathbb{R} \times \mathcal{C} \rightarrow \mathbb{R}^{n}$ and $\mathcal{L}(\cdot, \cdot): \mathbb{R} \times \mathcal{C} \rightarrow \mathbb{R}^{n}$ are given continuous functions such that $\mathcal{D}(t, \cdot)$ and $\mathcal{L}(t, \cdot)$ are bounded linear operators from $\mathcal{C}$ to $\mathbb{R}^{n}$ for each $t \in \mathbb{R}$.

In general, it is not easy to tackle the problem of stability analysis of time-varying differential equations of neutral type. The traditional approaches to problems of stability of time-varying differential equations of neutral type are based on the Lyapunov's method and its variants, see e.g. [4-[10], [12]-[16], [18], 19]. Most existing results in the literature are given in terms of matrix inequalities and not straightforward to use.

There have been many works dedicated to stability of differential equations of neutral type, see [1]-[2], [4]-[10], [12]-[20]. However, problems of exponential stability of the linear neutral functional differential equation (1) has not yet been studied adequately and to

1991 Mathematics Subject Classification. 34K20.

Key words and phrases. Linear functional differential equation, neutral equation, exponential stability 
date there have been no explicit stability criteria for (1) in the literature. The main purpose of this paper is to fill this gap.

In contrast to the traditional approaches, we present in this paper a novel approach to exponential stability of the linear neutral functional differential equation (1). Our approach is simple in that it relies only upon a system transformation, the comparison principle and spectral properties of Metzler matrices. Consequently, we can obtain new explicit criteria for exponential stability of (1). A discussion to the obtained results and a numerial example are given for illustration.

The organization of the paper is as follows. In the next section, we give notation and preliminary results to be used in our development. The main results are presented in Section 3. We derive new explicit criteria for exponential stability of the linear neutral functional differential equation (1). In Section 4, we make a comparison of the obtained results with some existing ones in the literature and give an illustrative example. Finally, a conclusion is drawn in Section 5 .

\section{PRELIMINARIES}

Let $\mathbb{N}$ be the set of all natural numbers. For given $m \in \mathbb{N}$, let $\underline{m}:=\{1,2, \ldots, m\}$. For given integers $l, q \geq 1, \mathbb{R}^{l}$ denotes the $l$-dimensional vector space over $\mathbb{R}$ and $\mathbb{R}^{l \times q}$ stands for the set of all $l \times q$-matrices with entries in $\mathbb{R}$. For $A=\left(a_{i j}\right) \in \mathbb{R}^{l \times q}$ and $B=\left(b_{i j}\right) \in \mathbb{R}^{l \times q}, A \geq B$ means that $a_{i j} \geq b_{i j}$ for $i=1, \cdots, l, j=1, \cdots, q$. In particular, if $a_{i j}>b_{i j}$ for $i=1, \cdots, l, j=1, \cdots, q$, then we write $A \gg B$ instead of $A \geq B$. Denote by $\mathbb{R}_{+}^{l \times q}$ the set of all nonnegative matrices. Similar notations are adopted for vectors.

For $x \in \mathbb{R}^{n}$ and $P \in \mathbb{R}^{l \times q}$ we define $|x|=\left(\left|x_{i}\right|\right)$ and $|P|=\left(\left|p_{i j}\right|\right)$. Then one has

$$
|P Q| \leq|P||Q|, \quad \forall P \in \mathbb{R}^{l \times q}, \quad \forall Q \in \mathbb{R}^{q \times r} .
$$

Let $I_{n}$ be the identity matrix in $\mathbb{R}^{n \times n}$. For any matrix $M \in \mathbb{R}^{n \times n}$ the spectral abscissa (resp. the spectral radius) of $M$ is defined by $s(M):=\max \{\Re \lambda: \lambda \in \sigma(M)\}$ (resp. $\rho(M):=\max \{|\lambda|: \lambda \in \sigma(M)\})$ where $\sigma(M):=\left\{z \in \mathbb{C}: \operatorname{det}\left(z I_{n}-M\right)=0\right\}$ is the spectrum of $M$. A matrix $M \in \mathbb{R}^{n \times n}$ is said to be Hurwitz stable (resp. Schur stable) if, $s(M)<0$ (resp. $\rho(M)<1$ ). A norm $\|\cdot\|$ on $\mathbb{R}^{n}$ is said to be monotonic if $\|x\| \leq\|y\|$ whenever $x, y \in \mathbb{R}^{n},|x| \leq|y|$. Every $p$-norm on $\mathbb{R}^{n}$

$$
\|x\|_{p}=\left(\left|x_{1}\right|^{p}+\left|x_{2}\right|^{p}+\ldots+\left|x_{n}\right|^{p}\right)^{\frac{1}{p}}, \quad 1 \leq p<\infty ; \quad\|x\|_{\infty}=\max _{i=1,2, \ldots, n}\left|x_{i}\right|,
$$

is monotonic. Throughout the paper, if otherwise not stated, the norm of vectors on $\mathbb{R}^{n}$ is monotonic and the norm of a matrix $P \in \mathbb{R}^{l \times q}$ is understood as its operator norm associated with a given pair of monotonic vector norms on $\mathbb{R}^{l}$ and $\mathbb{R}^{q}$, that is

$$
\|P\|=\max \{\|P y\|: \quad\|y\|=1\}
$$


A matrix $M \in \mathbb{R}^{n \times n}$ is called a Metzler matrix if all off-diagonal elements of $M$ are nonnegative. For given $A:=\left(a_{i j}\right) \in \mathbb{R}^{n \times n}$, we associate the Metzler matrix $M(A):=\left(\hat{a}_{i j}\right)$ where $\hat{a}_{i j}=\left|a_{i j}\right|$ if $i \neq j$, for $i, j \in \underline{n}$ and $\hat{a}_{i i}=a_{i i}$, for $i \in \underline{n}$.

Theorem 2.1. 3

(i) Let $A \in \mathbb{R}_{+}^{n \times n}$. Then $A$ is Schur stable if and only if $A q \ll q$, for some $q \in \mathbb{R}_{+}^{n}, q \gg 0$.

(ii) Let $A \in \mathbb{R}^{n \times n}$ be a Metzler matrix. Then $A$ is Hurwitz stable if and only if $A p \ll 0$, for some $p \in \mathbb{R}_{+}^{n}, p \gg 0$.

The following lemma is used in what follows.

Lemma 2.2. Let $A \in \mathbb{R}^{n \times n}$ be a Metzler matrix and $B, C, D \in \mathbb{R}_{+}^{n \times n}$. Then the following statements are equivalent

(i) $\rho(D)<1$ and $s\left(A+B\left(I_{n}-D\right)^{-1} C\right)<0$;

(ii) there exist $p, q \in \mathbb{R}_{+}^{n}, p \gg 0, q \gg 0$ such that

$$
\left(\begin{array}{ll}
A & B \\
C & D
\end{array}\right)\left(\begin{array}{l}
p \\
q
\end{array}\right) \ll\left(\begin{array}{l}
0 \\
q
\end{array}\right) .
$$

(iii) $s(A)<0$ and $\rho\left(C(-A)^{-1} B+D\right)<1$.

Proof. (i) $\Rightarrow$ (ii) Since $D \in \mathbb{R}_{+}^{n \times n}$ and $\rho(D)<1$, it follows that $\left(I_{n}-D\right)^{-1}=\sum_{k=0}^{\infty} D^{k} \geq 0$. Furthermore, we have $D q_{0} \ll q_{0}$, for some $q_{0} \in \mathbb{R}_{+}^{n}, q_{0} \gg 0$, by Theorem 2.1 (i). Thus, $B\left(I_{n}-D\right)^{-1} C \in \mathbb{R}_{+}^{n \times n}$ and $A+B\left(I_{n}-D\right)^{-1} C$ is a Metzler matrix. Since $s\left(A+B\left(I_{n}-\right.\right.$ $\left.D)^{-1} C\right)<0$, it follows that $\left(A+B\left(I_{n}-D\right)^{-1} C\right) p \ll 0$ for some $p \in \mathbb{R}_{+}^{n}, p \gg 0$, by Theorem 2.1 (ii). This implies $\left(A+B\left(I_{n}-D\right)^{-1} C\right) p+\epsilon B q_{0} \ll 0$, for sufficiently small $\epsilon$. Let $q:=\left(I_{n}-D\right)^{-1} C p+\epsilon q_{0} \gg 0$. Note that $A p+B q=A p+\left(B\left(I_{n}-D\right)^{-1} C p+\epsilon B q_{0}\right) \ll 0$ and $C p+D q=q+\epsilon\left(D q_{0}-q_{0}\right) \ll q$. Therefore, (i) holds.

(ii) $\Rightarrow$ (i) Assume that $A p+B q \ll 0$ and $C p+D q \ll q$, with $p, p \in \mathbb{R}_{+}^{n}, p, q \gg 0$. Note that $D q \leq C p+D q \ll q$, with $q \in \mathbb{R}_{+}^{n}, q \gg 0$. Thus, $\rho(D)<1$, by Theorem 2.1 (i). Furthermore, $C p \ll q-D q$. It follows that $q=\left(I_{n}-D\right)^{-1}\left(I_{n}-D\right) q \geq\left(I_{n}-D\right)^{-1} C p$. Therefore, $0 \gg A p+B q \geq A p+B\left(I_{n}-D\right)^{-1} C p=\left(A+B\left(I_{n}-D\right)^{-1} C\right) p$. Thus, $s\left(A+B\left(I_{n}-D\right)^{-1} C\right)<0$, by Theorem 2.1 (ii).

(ii) $\Rightarrow$ (iii) Suppose $A p+B q \ll 0$ and $C p+D q \ll q$, with $p, q \in \mathbb{R}_{+}^{n}, p, q \gg 0$. From $B \in \mathbb{R}_{+}^{n \times n}$ and $q \in \mathbb{R}_{+}^{n}$, it follows that $B q \in \mathbb{R}_{+}^{n}$. Thus, $A p \leq A p+B q \ll 0$. Therefore, $s(A)<0$, by Theorem 2.1 (ii). Since $A$ is a Metzler matrix and $s(A)<0$, one has $(-A)^{-1} \in \mathbb{R}_{+}^{n \times n}$, see e.g. [3]. From $A p+B q \ll 0$, it follows that $B q \ll-A p$. This gives $(-A)^{-1} B q \leq p$ and $C(-A)^{-1} B q \leq C p$. Thus, $\left(C(-A)^{-1} B+D\right) q \leq C p+D q \ll q$. By Theorem 2.1 (i), $\rho\left(C(-A)^{-1} B+D\right)<1$.

(iii) $\Rightarrow$ (ii) Assume that $s(A)<0$ and $\rho\left(C(-A)^{-1} B+D\right)<1$. As mentioned above, it follows from $s(A)<0$ that $(-A)^{-1} \in \mathbb{R}_{+}^{n \times n}$. By Theorem 2.1, $A p_{0} \ll 0$ and $\left(C(-A)^{-1} B+\right.$ $D) q \ll q$, for some $p_{0}, q \in \mathbb{R}_{+}^{n}, p_{0}, q \gg 0$. This implies, $\left(C(-A)^{-1} B+D\right) q+\gamma C p_{0} \ll q$, 
for sufficiently small $\gamma>0$. Let $p:=(-A)^{-1} B q+\gamma p_{0} \gg 0$. Then, we have $C p+D q=$ $\left(C(-A)^{-1} B+D\right) q+\gamma C p_{0} \ll q$ and $A p+B q=A\left((-A)^{-1} B q+\gamma p_{0}\right)+B q=\gamma A p_{0} \ll 0$. This completes the proof.

To make a representation self contained and dynamic, we present here some basic facts on vector-valued functions of bounded variation and related topics. A matrix function $\eta(\cdot):[\alpha, \beta] \rightarrow \mathbb{R}^{m \times n}$ is called an increasing matrix function if

$$
\eta\left(\theta_{2}\right) \geq \eta\left(\theta_{1}\right) \quad \text { for } \alpha \leq \theta_{1} \leq \theta_{2} \leq \beta
$$

A matrix-valued function $\eta(\cdot):[\alpha, \beta] \rightarrow \mathbb{R}^{m \times n}$ is said to be of bounded variation if

$$
\operatorname{Var}_{[\alpha, \beta]} \eta(\cdot):=\sup _{P[\alpha, \beta]} \sum_{k}\left\|\eta\left(\theta_{k}\right)-\eta\left(\theta_{k-1}\right)\right\|<+\infty,
$$

where the supremum is taken over the set of all finite partitions of the interval $[\alpha, \beta]$. The set $B V\left([\alpha, \beta], \mathbb{R}^{m \times n}\right)$ of all matrix functions $\eta(\cdot)$ of bounded variation on $[\alpha, \beta]$ satisfying $\eta(\alpha)=0$ is a Banach space endowed with the norm $\|\eta\|=\operatorname{Var}_{[\alpha, \beta]} \eta(\cdot)$. Since all matrix norms on $\mathbb{R}^{m \times n}$ are equivalent, it follows that the matrix function $\eta(\cdot)=\left(\eta_{i j}(\cdot)\right) \in \mathbb{R}^{m \times n}$ is of bounded variation if and only if each $\eta_{i j}(\cdot)$ is of bounded variation. Then $\eta(\cdot) \in$ $B V\left([\alpha, \beta], \mathbb{R}^{m \times n}\right)$, if and only if

$$
\eta(\cdot)=\eta_{2}(\cdot)-\eta_{1}(\cdot)
$$

where $\eta_{1}(\cdot)$ and $\eta_{2}(\cdot)$ are increasing matrix functions, see e.g. [11]. Therefore, $\lim _{\theta \rightarrow \theta_{0}^{-}} \eta(\theta)$ exists for any $\theta_{0} \in[\alpha, \beta]$, if $\eta(\cdot) \in B V\left([\alpha, \beta], \mathbb{R}^{m \times n}\right)$.

Let us consider a subset of $B V\left([\alpha, \beta], \mathbb{R}^{m \times n}\right)$ :

$$
N B V\left([\alpha, \beta], \mathbb{R}^{m \times n}\right):=\left\{\eta \in B V\left([\alpha, \beta], \mathbb{R}^{m \times n}\right) ; \eta \text { is continuous from left on }(\alpha, \beta)\right\} \text {. }
$$

Clearly, $N B V\left([\alpha, \beta], \mathbb{R}^{m \times n}\right)$ is closed in $B V\left([\alpha, \beta], \mathbb{R}^{m \times n}\right)$ and thus it is a Banach space with the norm $\|\eta\|=\operatorname{Var}_{[\alpha, \beta]} \eta(\cdot)$. Given $\eta(\cdot) \in N B V\left([\alpha, \beta], \mathbb{R}^{m \times n}\right)$ then for any continuous functions $\gamma \in C([\alpha, \beta], \mathbb{R})$ and $\varphi \in C\left([\alpha, \beta], \mathbb{R}^{n}\right)$, the integrals

$$
\int_{\alpha}^{\beta} \gamma(\theta) d[\eta(\theta)] \text { and } \int_{\alpha}^{\beta} d[\eta(\theta)] \varphi(\theta)
$$

exist and are defined respectively as the limits of $S_{1}(P):=\sum_{k=1}^{p} \gamma\left(\zeta_{k}\right)\left(\eta\left(\theta_{k}\right)-\eta\left(\theta_{k-1}\right)\right)$ and $S_{2}(P):=\sum_{k=1}^{p}\left(\eta\left(\theta_{k}\right)-\eta\left(\theta_{k-1}\right)\right) \varphi\left(\zeta_{k}\right)$ when $d(P):=\max _{k}\left|\theta_{k}-\theta_{k-1}\right| \rightarrow 0$, where $P=\left\{\theta_{1}=\alpha \leq \theta_{2} \leq \cdots \leq \theta_{p}=\beta\right\}$ is any finite partition of the interval $[\alpha, \beta]$ and $\zeta_{k} \in\left[\theta_{k-1}, \theta_{k}\right]$. It is immediate from the definition that

$$
\begin{aligned}
\left\|\int_{\alpha}^{\beta} \gamma(\theta) d[\eta(\theta)]\right\| & \leq \max _{\theta \in[\alpha, \beta]}|\gamma(\theta)|\|\eta\|, \\
\left\|\int_{\alpha}^{\beta} d[\eta(\theta)] \varphi(\theta)\right\| & \leq \max _{\theta \in[\alpha, \beta]}\|\varphi(\theta)\|\|\eta\| .
\end{aligned}
$$


Let $\mathbb{R}^{m \times n}$ be endowed with the norm $\|\cdot\|$ and let $J$ be an interval of $\mathbb{R}$. Denote by $C\left(J, \mathbb{R}^{m \times n}\right)$, the vector space of all continuous functions on $J$ with values in $\mathbb{R}^{m \times n}$. In particular, $C\left([\alpha, \beta], \mathbb{R}^{m \times n}\right)$ is a Banach space endowed with the norm

$$
\|\varphi\|:=\max _{\theta \in[\alpha, \beta]}\|\varphi(\theta)\|
$$

In the following, the Banach space $C\left([-h, 0], \mathbb{R}^{n}\right)$ is used frequently. For simplicity, we write $\mathcal{C}$ instead of $C\left([-h, 0], \mathbb{R}^{n}\right)$.

\section{EXPLICIT CRITERIA FOR EXPONENTIAL STABILITY}

Consider the linear neutral functional differential equation (1), where $\mathcal{D}(\cdot, \cdot): \mathbb{R} \times \mathcal{C} \rightarrow$ $\mathbb{R}^{n}$ and $\mathcal{L}(\cdot, \cdot): \mathbb{R} \times \mathcal{C} \rightarrow \mathbb{R}^{n}$ are given continuous functions such that $\mathcal{D}(t, \cdot)$ and $\mathcal{L}(t, \cdot)$ are bounded linear operators from $\mathcal{C}$ to $\mathbb{R}^{n}$ for each $t \in \mathbb{R}$.

Throughout, we assume that

$$
\mathcal{D}(t, \varphi):=\varphi(0)-\mathcal{D}_{0}(t, \varphi), \quad t \in \mathbb{R}
$$

and $\mathcal{D}_{0}(t, \cdot)$ is a bounded linear operator from $\mathcal{C}$ to $\mathbb{R}^{n}$ for each $t \in \mathbb{R}$.

By the well-known Riesz representation theorem,

$$
\mathcal{L}(t, \varphi):=\int_{-h}^{0} d[\eta(t, \theta)] \varphi(\theta), \quad \varphi \in \mathcal{C}, \quad t \in \mathbb{R}
$$

and

$$
\mathcal{D}_{0}(t, \varphi):=\int_{-h}^{0} d[\mu(t, \theta)] \varphi(\theta), \quad \varphi \in \mathcal{C}, \quad \forall t \in \mathbb{R},
$$

where for each $t \in \mathbb{R}, \eta(t, \theta): \mathbb{R} \times[-h, 0] \rightarrow \mathbb{R}^{n \times n}$ and $\mu(t, \theta): \mathbb{R} \times[-h, 0] \rightarrow \mathbb{R}^{n \times n}$ are of bounded variation in $\theta$ on $[-h, 0]$ and normalized so that $\eta(t, \theta)$ and $\mu(t, \theta)$ are continuous from the left in $\theta$ on $(-h, 0)$ and

$$
\begin{aligned}
& \eta(t, \theta)=0, \quad \theta \geq 0, \quad \eta(t, \theta)=\eta(t,-h), \quad \forall \theta \leq-h, \\
& \mu(t, \theta)=0, \quad \theta \geq 0, \quad \mu(t, \theta)=\mu(t,-h), \quad \forall \theta \leq-h .
\end{aligned}
$$

Furthermore, suppose there exists a constant matrix $D:=\left(d_{i j}\right) \in \mathbb{R}_{+}^{n \times n}$ such that

$$
\operatorname{Var}_{[-h, 0]} \mu_{i j}(t, \cdot) \leq d_{i j}, \quad \forall t \in \mathbb{R}, \forall i, j \in \underline{n}
$$

where $\mu(t, \theta):=\left(\mu_{i j}(t, \theta)\right) \in \mathbb{R}^{n \times n}$ for $t \in \mathbb{R}, \theta \in[-h, 0]$ and $\mu$ is uniformly nonatomic at zero, that is, for every $\epsilon>0$, there exists $\delta>0$ such that

$$
\operatorname{Var}_{[-\delta, 0]} \mu(t, \cdot)<\epsilon, \quad \forall t \in \mathbb{R} .
$$


Remark 3.1. (i) Suppose $\mu(t, \cdot), t \in \mathbb{R}$ has no singular part, that is

$$
\int_{-h}^{0} d[\mu(t, \theta)] \varphi(\theta):=\sum_{i=1}^{m} D_{i}(t) \varphi\left(-h_{i}\right)+\int_{-h}^{0} E(t, s) \varphi(\theta) d s, \quad \varphi \in \mathcal{C},
$$

where $0 \leq h_{i} \leq h, i \in \underline{m}$ and $D_{i}(\cdot): \mathbb{R} \rightarrow \mathbb{R}^{n \times n}, i \in \underline{m}, E(\cdot, \cdot): \mathbb{R} \times[-h, 0] \rightarrow \mathbb{R}^{n \times n}$, are continuous functions. Furthermore, assume that there exists $D_{i} \in \mathbb{R}_{+}^{n \times n}, i \in \underline{m}$ and a continuous function $E_{0}(\cdot):[-h, 0] \rightarrow \mathbb{R}_{+}^{n \times n}$ such that

$$
\left|D_{i}(t)\right| \leq D_{i}, \forall t \in \mathbb{R}, \forall i \in \underline{m} ; \quad|E(t, s)| \leq E_{0}(s), \forall t \in \mathbb{R}, \forall s \in[-h, 0] .
$$

Clearly, (6) holds and $\mu$ is uniformly nonatomic at zero.

(ii) Assume that $\mu(t, \cdot) \equiv \mu(\cdot)$, i.e., independent of $t$. Then $\mu(\cdot)$ is uniformly nonatomic at zero if $\operatorname{Var}_{[-s, 0]} \mu(\cdot) \rightarrow 0$ as $s \rightarrow 0$.

For given $\varphi \in \mathcal{C}$, consider for (1) the initial condition

$$
x(\theta+\sigma)=\varphi(\theta), \quad \theta \in[-h, 0] .
$$

Definition 3.2. Let $\sigma \in \mathbb{R}$ and $\varphi \in \mathcal{C}$ be given. A continuous function $x(\cdot):[-h+$ $\sigma, \infty) \rightarrow \mathbb{R}^{n}$, is said to be a solution of (1) with the initial value $(\sigma, \varphi)$ (or simply, a solution of (1) through $(\sigma, \varphi))$ if $\mathcal{D}\left(t, x_{t}\right)$ is continuously differentiable on $(\sigma, \infty)$ with a right hand derivative at $\sigma$ and $x(\cdot)$ satisfies (1) on $[\sigma, \infty)$ and satisfies (10) on $[-h, 0]$.

It is well-known that for fixed $\sigma \in \mathbb{R}$ and given $\varphi \in \mathcal{C}$, there exists a unique solution of (1) through $(\sigma, \varphi)$, denoted by $x(\cdot ; \sigma, \varphi)$, provided (6) holds and $\mu$ is uniformly nonatomic at zero, see e.g. [9, Theorem 1.1, page 256]

Definition 3.3. The equation (1) is said to be exponentially stable if there exist $K, \beta>0$ such that

$$
\|x(t ; \sigma, \varphi)\| \leq K e^{-\beta(t-\sigma)}\|\varphi\|, \quad \forall t \geq \sigma, \forall \varphi \in \mathcal{C} .
$$

Let $\hat{\eta}(t, \theta):=\eta(t, \theta), \theta \in[-h, 0) ; \hat{\eta}(t, 0):=\lim _{\theta \rightarrow 0^{-}} \eta(t, \theta)$ and let $\tilde{\eta}(t, \theta):=0, \theta \in$ $[-h, 0) ; \tilde{\eta}(t, 0):=\eta(t, 0)-\lim _{\theta \rightarrow 0^{-}} \eta(t, \theta)=-\lim _{\theta \rightarrow 0-} \eta(t, \theta):=A(t)$. Clearly,

$$
\eta(t, \theta)=\hat{\eta}(t, \theta)+\tilde{\eta}(t, \theta), \quad \theta \in[-h, 0],
$$

and $\hat{\eta}(t, \cdot)$ is continuous from the left on $[-h, 0]$. Therefore,

$$
\mathcal{L}(t, \varphi):=\int_{-h}^{0} d[\eta(t, \theta)] \varphi(\theta)=A(t) \varphi(0)+\int_{-h}^{0} d[\hat{\eta}(t, \theta)] \varphi(\theta), \quad t \in \mathbb{R}, \varphi \in \mathcal{C},
$$

and thus (1) is reduced to

$$
\frac{d}{d t}\left(x(t)-\int_{-h}^{0} d[\mu(t, \theta)] x(t+\theta)\right)=A(t) x(t)+\int_{-h}^{0} d[\hat{\eta}(t, \theta)] x(t+\theta), \quad t \geq \sigma .
$$

In addition, we always assume that $A(\cdot): \mathbb{R} \rightarrow \mathbb{R}^{n \times n}$ and $\mathcal{L}_{1}(\cdot ; \cdot): \mathbb{R} \times \mathcal{C} \rightarrow \mathbb{R}^{n},(t, \varphi) \mapsto$ $\mathcal{L}_{1}(t ; \varphi):=\int_{-h}^{0} d[\hat{\eta}(t, \theta)] \varphi(\theta)$, are continuous functions.

We are now in the position to state the main result of this paper. 
Theorem 3.4. Let

$$
\bar{\mu}(t, \theta):=A(t) \mu(t, \theta), t \in \mathbb{R}, \theta \in[-h, 0] .
$$

Suppose there exist $\beta>0$ and $p:=\left(p_{1}, p_{2}, \ldots, p_{n}\right)^{T}, q:=\left(q_{1}, q_{2}, \ldots, q_{n}\right)^{T} \in \mathbb{R}_{+}^{n}, p, q \gg 0$ such that

$$
\begin{gathered}
\left(a_{i i}(t) p_{i}+\sum_{j=1, j \neq i}^{n}\left|a_{i j}(t)\right| p_{j}\right)+\sum_{j=1}^{n}\left(\operatorname{Var}_{[-h, 0]} \bar{\mu}_{i j}(t, \cdot)+\operatorname{Var}_{[-h, 0]} \hat{\eta}_{i j}(t, \cdot)\right) e^{\beta h} q_{j}<-\beta p_{i} \\
p_{i}+\sum_{j=1}^{n} \operatorname{Var}_{[-h, 0]} \mu_{i j}(t, \cdot) e^{\beta h} q_{j}<q_{i},
\end{gathered}
$$

for any $t \in \mathbb{R}$ and for any $i \in \underline{n}$. Then (1) is exponentially stable.

Proof. Let $x(\cdot):=x(\cdot ; \sigma, \varphi)$, be the unique solution of (1) through $(\sigma, \varphi)$. Define

$$
y(t):=\mathcal{D}\left(t, x_{t}\right)=x(t)-\int_{-h}^{0} d[\mu(t, \theta)] x(t+\theta) \quad t \geq \sigma .
$$

Then $x(\cdot)$ and $y(\cdot)$ satisfy the following system

$$
\begin{gathered}
\frac{d y(t)}{d t}=A(t) y(t)+\int_{-h}^{0} d[\bar{\mu}(t, \theta)] x(t+\theta)+\int_{-h}^{0} d[\hat{\eta}(t, \theta)] x(t+\theta), \quad t \geq \sigma \\
x(t)=y(t)+\int_{-h}^{0} d[\mu(t, \theta)] x(t+\theta), \quad t \geq \sigma .
\end{gathered}
$$

Choose $K>0$ such that $|\varphi(t)| \ll K q$ and $|\varphi(0)|+D|\varphi(t)| \ll K p$, for any $t \in[-h, 0]$ and for any $\varphi \in \mathcal{C},\|\varphi\| \leq 1$. Define $u(t):=K e^{-\beta(t-\sigma)} q, t \in[\sigma-h, \infty)$ and $v(t):=$ $K e^{-\beta(t-\sigma)} p, t \in[\sigma, \infty)$. It follows that $|x(t)| \ll u(t), \forall t \in[\sigma-h, \sigma]$ and $|y(\sigma)| \ll v(\sigma)$. We claim that $|x(t)| \leq u(t), \forall t \geq \sigma$ and $|y(t)| \leq v(t), \forall t \geq \sigma$.

Assume on the contrary that there exists $t_{0}>\sigma$ such that either $\left|x\left(t_{0}\right)\right| \not \leq u\left(t_{0}\right)$ or $\left|y\left(t_{0}\right)\right| \not \leq v\left(t_{0}\right)$. Set $t_{1}:=\inf \{t \in(\sigma, \infty):(|x(t)|,|y(t)|) \not \leq(u(t), v(t))\}$. By continuity, $t_{1}>\sigma$ and one of the following statements holds:

(i) $|x(t)| \leq u(t), t \in\left[\sigma, t_{1}\right]$ and there is $i_{0} \in \underline{n}$ such that

$$
|y(t)| \leq v(t), \forall t \in\left[\sigma, t_{1}\right) ;\left|y_{i_{0}}\left(t_{1}\right)\right|=v_{i_{0}}\left(t_{1}\right),\left|y_{i_{0}}\left(\tau_{k}\right)\right|>v_{i_{0}}\left(\tau_{k}\right)
$$

for some $\tau_{k} \in\left(t_{1}, t_{1}+\frac{1}{k}\right), k \in \mathbb{N}$.

(ii) $|y(t)| \leq v(t), t \in\left[\sigma, t_{1}\right]$ and there is $k_{0} \in \underline{n}$ such that

$$
|x(t)| \leq u(t), \forall t \in\left[\sigma, t_{1}\right) ;\left|x_{k_{0}}\left(t_{1}\right)\right|=u_{k_{0}}\left(t_{1}\right),\left|x_{k_{0}}\left(\xi_{k}\right)\right|>u_{k_{0}}\left(\xi_{k}\right),
$$

for some $\xi_{k} \in\left(t_{1}, t_{1}+\frac{1}{k}\right), k \in \mathbb{N}$. 
Suppose (i) holds. Let $A(t):=\left(a_{i j}(t)\right) ; \bar{\mu}(t, \theta):=\left(\bar{\mu}_{i j}(t, \theta)\right) ; \hat{\eta}(t, \theta)=\left(\hat{\eta}_{i j}(t, \theta)\right), t \in$ $\mathbb{R}, \theta \in[-h, 0]$. It follows from $(16)$ that

$$
\begin{gathered}
\frac{d}{d t}\left|y_{i}(t)\right|=\operatorname{sgn}\left(y_{i}(t)\right) \dot{y}_{i}(t) \leq a_{i i}(t)\left|y_{i}(t)\right|+\sum_{j=1, j \neq i}^{n}\left|a_{i j}(t)\right|\left|y_{j}(t)\right|+ \\
\left|\sum_{j=1}^{n} \int_{-h}^{0} d\left[\bar{\mu}_{i j}(t, \theta)\right] x_{j}(t+\theta)\right|+\left|\sum_{j=1}^{n} \int_{-h}^{0} d\left[\hat{\eta}_{i j}(t, \theta)\right] x_{j}(t+\theta)\right|,
\end{gathered}
$$

for almost any $t \in[\sigma,+\infty)$ and any $i \in \underline{n}$. It follows that for any $t \in[\sigma,+\infty)$

$$
\begin{aligned}
D^{+}\left|y_{i}(t)\right|:= & \limsup _{h \rightarrow 0^{+}} \frac{\left|y_{i}(t+h)\right|-\left|y_{i}(t)\right|}{h}=\limsup _{h \rightarrow 0^{+}} \frac{1}{h} \int_{t}^{t+h} \frac{d}{d \zeta}\left|y_{i}(\zeta)\right| d \zeta \\
& \leq a_{i i}(t)\left|y_{i}(t)\right|+\sum_{j=1, j \neq i}^{n}\left|a_{i j}(t)\right|\left|y_{j}(t)\right|+ \\
& \sum_{j=1}^{n}\left|\int_{-h}^{0} d\left[\bar{\mu}_{i j}(t, \theta)\right] x_{j}(t+\theta)\right|+\sum_{j=1}^{n}\left|\int_{-h}^{0} d\left[\hat{\eta}_{i j}(t, \theta)\right] x_{j}(t+\theta)\right|,
\end{aligned}
$$

where $D^{+}$denotes the Dini upper-right derivative. In particular, it follows from (i) that

$$
\begin{aligned}
D^{+}\left|y_{i_{0}}\left(t_{1}\right)\right| \leq & a_{i_{0} i_{0}}\left(t_{1}\right) K e^{-\beta\left(t_{1}-\sigma\right)} p_{i_{0}}+\sum_{j=1, j \neq i_{0}}^{n}\left|a_{i_{0} j}\left(t_{1}\right)\right| K e^{-\beta\left(t_{1}-\sigma\right)} p_{j}+ \\
& \sum_{j=1}^{n}\left(\operatorname{Var}_{[-h, 0]} \bar{\mu}_{i_{0} j}\left(t_{1}, \cdot\right)+\sum_{j=1}^{n} \operatorname{Var}_{[-h, 0]} \hat{\eta}_{i_{0} j}\left(t_{1}, \cdot\right)\right) K e^{-\beta\left(t_{1}-\sigma\right)} e^{\beta h} q_{j} \\
& =K e^{-\beta\left(t_{1}-\sigma\right)}\left(a_{i_{0} i_{0}}\left(t_{1}\right) p_{i_{0}}+\sum_{j=1, j \neq i_{0}}^{n}\left|a_{i_{0} j}\left(t_{1}\right)\right| p_{j}+\right. \\
& \left.\sum_{j=1}^{n}\left(\operatorname{Var}_{[-h, 0]} \bar{\mu}_{i_{0} j}\left(t_{1}, \cdot\right)+\sum_{j=1}^{n} \operatorname{Var}_{[-h, 0]} \hat{\eta}_{i_{0} j}\left(t_{1}, \cdot\right)\right) e^{\beta h} q_{j}\right) \\
& \stackrel{14}{<}-\beta K e^{-\beta\left(t_{1}-\sigma\right)} p_{i_{0}}=D^{+} v_{i_{0}}\left(t_{1}\right) .
\end{aligned}
$$

On the other hand, (18) implies that

$$
\begin{aligned}
& D^{+}\left|y_{i_{0}}\left(t_{1}\right)\right|=\limsup _{t \rightarrow t_{1}^{+}} \frac{\left|y_{i_{0}}(t)\right|-\left|y_{i_{0}}\left(t_{1}\right)\right|}{t-t_{1}} \geq \varlimsup_{k \rightarrow \infty} \frac{\left|y_{i_{0}}\left(\tau_{k}\right)\right|-\left|y_{i_{0}}\left(t_{1}\right)\right|}{\tau_{k}-t_{1}} \\
& \geq \varlimsup_{k \rightarrow \infty} \frac{v_{i_{0}}\left(\tau_{k}\right)-v_{i_{0}}\left(t_{1}\right)}{\tau_{k}-t_{1}}=\lim _{k \rightarrow \infty} \frac{v_{i_{0}}\left(\tau_{k}\right)-v_{i_{0}}\left(t_{1}\right)}{\tau_{k}-t_{1}}=\frac{d v_{i_{0}}}{d t}\left(t_{1}\right)=D^{+} v_{i_{0}}\left(t_{1}\right) .
\end{aligned}
$$

This is a contradition.

Assume that (ii) holds. It follows from (17) and (19) that

$$
\left|x_{k_{0}}\left(t_{1}\right)\right| \stackrel{\sqrt[17]{\leq}}{\leq}\left|y_{k_{0}}\left(t_{1}\right)\right|+\sum_{j=1}^{n}\left|\int_{-h}^{0} d\left[\mu_{k_{0} j}\left(t_{1}, \theta\right)\right] x_{j}\left(t_{1}+\theta\right)\right|
$$




$$
\begin{gathered}
\leq\left|y_{k_{0}}\left(t_{1}\right)\right|+\sum_{j=1}^{n} \operatorname{Var}_{[-h, 0]} \mu_{k_{0} j}\left(t_{1}, \theta\right) \sup _{\theta \in[-h, 0]}\left|x_{j}\left(t_{1}+\theta\right)\right| \\
\stackrel{(i i)}{\leq} K e^{-\beta\left(t_{1}-\sigma\right)} p_{k_{0}}+\sum_{j=1}^{n} \operatorname{Var}_{[-h, 0]} \mu_{k_{0} j}\left(t_{1}, \theta\right) K e^{-\beta\left(t_{1}-\sigma\right)} q_{j} e^{\beta h} \\
=K e^{-\beta\left(t_{1}-\sigma\right)}\left(p_{k_{0}}+\sum_{j=1}^{n} \operatorname{Var}_{[-h, 0]} \mu_{k_{0} j}\left(t_{1}, \theta\right) e^{\beta h} q_{j}\right) \\
\stackrel{\text { (15) }}{<} K e^{-\beta\left(t_{1}-\sigma\right)} q_{k_{0}}=u_{k_{0}}\left(t_{1}\right) .
\end{gathered}
$$

This conflicts with (19). Thus, we have $|x(t)| \leq u(t), \forall t \geq \sigma$ and $|y(t)| \leq v(t), \forall t \geq \sigma$. In particular, this yields $|x(t ; \sigma, \varphi)| \leq K e^{-\beta(t-\sigma)} q, \forall t \geq \sigma, \forall \varphi \in \mathcal{C},\|\varphi\| \leq 1$. Since (1) is linear, it follows that

$$
\left|x\left(t ; \sigma, \frac{\varphi}{\|\varphi\|}\right)\right|=\frac{1}{\|\varphi\|}|x(t ; \sigma, \varphi)| \leq K e^{-\beta(t-\sigma)} q, \quad \forall t \geq \sigma, \forall \varphi \in \mathcal{C}, \varphi \neq 0 .
$$

Therefore,

$$
|x(t ; \sigma, \varphi)| \leq K\|\varphi\| e^{-\beta\left(t_{1}-\sigma\right)} q, \forall t \geq \sigma, \forall \varphi \in \mathcal{C}
$$

This yields

$$
\|x(t ; \sigma, \varphi)\| \leq K\|q\| e^{-\beta\left(t_{1}-\sigma\right)}\|\varphi\|, \forall t \geq \sigma, \forall \varphi \in \mathcal{C} .
$$

So (1) is exponentially stable. This completes the proof.

Remark 3.5. (i) Let

$$
V(t):=\left(V_{i j}(t)\right) \in \mathbb{R}_{+}^{n \times n}, t \in \mathbb{R},
$$

where

$$
V_{i j}(t):=\operatorname{Var}_{[-h, 0]} \bar{\mu}_{i j}(t, \cdot)+\operatorname{Var}_{[-h, 0]} \hat{\eta}_{i j}(t, \cdot), \quad t \in \mathbb{R} .
$$

Note that (14) and (15) can be represented in the form

$$
\begin{gathered}
(M(A(t))) p+V(t) e^{\beta h} q \ll-\beta p \\
p+\left(\operatorname{Var}_{[-h, 0]} \mu(t, \cdot)\right) e^{\beta h} q \ll q .
\end{gathered}
$$

(ii) The proof of Theorem 3.4 also shows that $\mathcal{D}(t, \varphi)$ exponentially decays. That is, there exist $K_{1}, \beta>0$ such that

$$
\|\mathcal{D}(t, \varphi)\| \leq K_{1} e^{-\beta(t-\sigma)}\|\varphi\|, \forall t \geq \sigma, \forall \varphi \in \mathcal{C} .
$$

Theorem 3.6. Let $D \in \mathbb{R}_{+}^{n \times n}$ satisfy (6) and let $V(t)$ be defined by (20). Suppose there exist $A \in \mathbb{R}^{n \times n}$ and $B \in \mathbb{R}_{+}^{n \times n}$ such that

$$
M(A(t)) \leq A, \forall t \in \mathbb{R} ; \quad V(t) \leq B, \forall t \in \mathbb{R} .
$$

Then (1) is exponentially stable if one of the following conditions holds: 
(i) there exist $\beta>0$ and $p, q \in \mathbb{R}_{+}^{n}, p \gg 0, q \gg 0$ such that

$$
A p+B e^{\beta h} q \ll-\beta p ; \quad p+D e^{\beta h} q \ll q .
$$

(ii) $\rho(D)<1$ and $s\left(A+B\left(I_{n}-D\right)^{-1}\right)<0$;

(iii) $s(A)<0$ and $\rho\left((-A)^{-1} B+D\right)<1$.

(iv) there exist $p, q \in \mathbb{R}_{+}^{n}, p \gg 0, q \gg 0$ such that

$$
\left(\begin{array}{ll}
A & B \\
I_{n} & D
\end{array}\right)\left(\begin{array}{l}
p \\
q
\end{array}\right) \ll\left(\begin{array}{l}
0 \\
q
\end{array}\right)
$$

Proof. Note that (ii), (iii) and (iv) of Theorem 3.6 are equivalent, by Lemma 2.2. By continuity, (iv) implies that (i) holds for sufficiently small $\beta>0$. Thus, it remains to show that (1) is exponentially stable provided (i) holds.

Clearly, (14)-(15) follow from $(23)$ and (31). Therefore, (1) is exponentially stable, by Theorem (3.4). This completes the proof.

Consider the linear neutral differential system

$$
\frac{d}{d t}\left(x(t)-\int_{-h}^{0} d[\mu(t, \theta)] x(t+\theta)\right)=A(t) x(t)+\sum_{k=1}^{r} B_{k}(t) x\left(t-\tau_{k}\right)+\int_{-h}^{0} C(t, \theta) x(t+\theta) d \theta, \quad t \geq \sigma,
$$

where $\mu(t, \theta)$ satisfies (8)-(9) and $A(\cdot): \mathbb{R} \rightarrow \mathbb{R}^{n \times n}, B_{k}(\cdot): \mathbb{R} \rightarrow \mathbb{R}^{n \times n}, k \in \underline{r}$ and $C(\cdot, \cdot)$ : $\mathbb{R} \times[-h, 0] \rightarrow \mathbb{R}^{n \times n}$, are given continuous functions and $0 \leq \tau_{k} \leq h, k \in \underline{r}$.

Let

$$
V_{0}(t):=\sum_{i=1}^{m}\left|A(t) D_{i}(t)\right|+\int_{-h}^{0}|A(t) E(t, s)| d s+\sum_{i=1}^{r}\left|B_{k}(t)\right|+\int_{-h}^{0}|C(t, s)| d s, \quad t \in \mathbb{R}
$$

The following theorems are immediate from Theorem 3.4 .

Theorem 3.7. Suppose there exist $\beta>0$ and $p, q \in \mathbb{R}_{+}^{n}, p, q \gg 0$ such that

$$
\begin{gathered}
(M(A(t))) p+V_{0}(t) e^{\beta h} q \ll-\beta p, \quad \forall t \in \mathbb{R}, \\
p+\left(\sum_{i=1}^{m}\left|D_{i}(t)\right|+\int_{-h}^{0}|E(t, s)| d s\right) e^{\beta h} q \ll q, \quad \forall t \in \mathbb{R} .
\end{gathered}
$$

Then (26) is exponentially stable.

Theorem 3.8. Let $D_{i} \in \mathbb{R}_{+}^{n \times n}(i \in \underline{m})$ and $E_{0}(\cdot):[-h, 0] \rightarrow \mathbb{R}_{+}^{n \times n}$ satisfy (9). Assume that there exist $A, B \in \mathbb{R}^{n \times n}$ such that

$$
M(A(t)) \leq A, t \in \mathbb{R} ; \quad V_{0}(t) \leq B, t \in \mathbb{R}
$$

Then (26) is exponentially stable if one of the following conditions holds: 
(i) there exist $\beta>0$ and $p, q \in \mathbb{R}_{+}^{n}, p \gg 0, q \gg 0$ such that

$$
A p+B e^{\beta h} q \ll-\beta p ; \quad p+\left(\sum_{i=1}^{m} D_{i}+\int_{-h}^{0} E_{0}(s) \mid d s\right) e^{\beta h} q \ll q ;
$$

(ii) $\rho\left(\sum_{i=1}^{m} D_{i}+\int_{-h}^{0} E_{0}(s) d s\right)<1$ and $s\left(A+B\left(I_{n}-\sum_{i=1}^{m} D_{i}-\int_{-h}^{0} E_{0}(s) d s\right)^{-1}\right)<0$;

(iii) $s(A)<0$ and $\rho\left((-A)^{-1} B+\sum_{i=1}^{m} D_{i}+\int_{-h}^{0} E_{0}(s) d s\right)<1$;

(iv) there exist $p, q \in \mathbb{R}_{+}^{n}, p \gg 0, q \gg 0$ such that

$$
\left(\begin{array}{cc}
A & B \\
I_{n} & \sum_{i=1}^{m} D_{i}+\int_{-h}^{0} E_{0}(s) d s
\end{array}\right)\left(\begin{array}{l}
p \\
q
\end{array}\right) \ll\left(\begin{array}{l}
0 \\
q
\end{array}\right) .
$$

Corollary 3.9. Suppose $A, B, D \in \mathbb{R}^{n \times n}$ are given and $C(\cdot): \mathbb{R} \rightarrow \mathbb{R}^{n \times n}$ is a given continuous function. The linear neutral time-invariant differential system

$$
\frac{d}{d t}(x(t)-D x(t-r))=A x(t)+B x(t-\tau)+\int_{-h}^{0} C(\theta) x(t+\theta) d \theta,
$$

is exponentially stable if

$$
\rho(|D|)<1 ; \quad s\left(M(A)+\left(|A D|+|B|+\int_{-h}^{0}|C(\theta)| d \theta\right)\left(I_{n}-|D|\right)^{-1}\right)<0 .
$$

Corollary 3.10. The linear differential system of retarded type

$$
\frac{d}{d t} x(t)=A(t) x(t)+\int_{-h}^{0} d[\hat{\eta}(t, \theta)] x(t+\theta), \quad t \geq \sigma .
$$

is exponentially stable if there exists $p \in \mathbb{R}^{n}, p \gg 0$ such that

$$
(M(A(t))) p+\left(\operatorname{Var}_{[-h, 0]} \hat{\eta}_{i j}(t, \theta)\right) e^{\beta h} p \ll-\beta p
$$

Furthermore, if there exist $A \in \mathbb{R}^{n \times n}$ and $B=\left(b_{i j}\right) \in \mathbb{R}_{+}^{n \times n}$ such that

$$
M(A(t)) \leq A, \quad t \in \mathbb{R} ; \quad\left(\operatorname{Var}_{[-h, 0]} \hat{\eta}_{i j}(t, \cdot)\right) \leq B, \quad \forall t \in \mathbb{R},
$$

and one of the following conditions holds:

(i) $s(A+B)<0$;

(ii) $s(A)<0$ and $\rho\left((-A)^{-1} B\right)<1$.

(iii) $(A+B) p \ll 0$ for some $p \in \mathbb{R}_{+}^{n}$.

then 35 is exponentially stable for any $h>0$.

\section{Discussion And illustrative examples}

As mentioned in the Introduction, problems of stability of linear neutral time-varying differential equations are not straightforward to solve. Up to our knowledge, there are no explicit criteria for exponential stability of linear neutral non-autonomous functional differential equations of the form (1). Some abstract criteria for asymptotic stability of (1) could be found in [9], [14] and recently in [8]. 
In [1], the authors considered the scalar linear neutral differential equation

$$
\frac{d}{d t}(x(t)-d(t) x(t-r))+a(t) x(t)+b(t) x(t-\tau)=0
$$

where $a(\cdot), b(\cdot), d(\cdot): \mathbb{R} \rightarrow \mathbb{R}_{+}$are continuous functions such that $p_{1} \leq a(t) \leq p_{2} ; q_{1} \leq$ $b(t) \leq q_{2}, 0 \leq d(t) \leq d_{1}<1, \forall t \in \mathbb{R}$ and $\left|d^{\prime}(t)\right| \leq d_{2}, \forall t \in \mathbb{R}$ for some $p_{1}, p_{2}, q_{1}, q_{2}, d_{1}, d_{2} \in$ $\mathbb{R}_{+}$. Using a Lyapunov functional, it has been shown in [1, Theorem 2] that if

$$
p_{1}>q_{2}+d_{1}\left(p_{2}+q_{2}\right)
$$

then every solution $x(t)$ of (38) satisfies $x(t) \rightarrow 0$ as $t \rightarrow \infty$. Some similar results can be found in [5], 9].

Note that $-a(t) \leq-p_{1}, \forall t \in \mathbb{R}$ and $|-b(t)| \leq q_{2}, \forall t \in \mathbb{R}$. By Theorem 3.8 (ii), (38) is exponentially stable if $-p_{1}+\left(p_{2} d_{1}+q_{2}\right)\left(1-d_{1}\right)^{-1}<0$, or equivalently,

$$
p_{1}>q_{2}+d_{1}\left(p_{1}+p_{2}\right) \text {. }
$$

Note that the conditions $\left|d^{\prime}(t)\right| \leq d_{2} ; a(t) \leq p_{2} ; b(t) \geq q_{1}, \forall t \in \mathbb{R}$, are redundant when we apply Theorem 3.8 (ii) to (38). Recall that, in general, exponential stability of linear neutral differential equations is stronger than their asymptotic stability, see [13].

Next, we consider a neutral logistic equation of the form

$$
\frac{d u(t)}{d t}=\gamma u(t)\left[p\left(1-\frac{u(t)}{k}\right)+q\left(1-\frac{u(t-h)}{k}\right)+c \frac{d}{d t}\left(1-\frac{u(t-\tau)}{k}\right)\right],
$$

where $\gamma, p, q, c, k, h, \tau$ are positive numbers.

Using the change of variable, $y(t)=\ln \frac{u(t)}{k}$, it has been shown in [1] that if the linear equation

$$
\frac{d}{d t}(y(t)+\gamma c y(t-\tau))=-\gamma p y(t)-\gamma q y(t-h),
$$

is exponentially stable, then the steady state value $k$ of 39 is locally asymptotically stable. Applying Theorem 3.7 to 40 , we conclude that 40 is exponentially stable if

$$
\gamma c<\frac{p-q}{2 p}
$$

To illustrate the advantage of the results of this paper, we present a numerical example for which the stability criteria given in [1], [7], [16], [18] cannot be applied.

Consider the linear time-varying differential system of neutral type defined by

$$
\begin{aligned}
& \dot{\zeta}(t)=-4 \zeta(t)-(\sin t) \zeta(t)-\frac{1}{t^{2}+16} \eta(t-1)+\frac{1}{16} e^{-t^{2}} \int_{-1}^{0} \eta(t+s) d s+\frac{1}{4} \dot{\zeta}(t-1) \\
& \dot{\eta}(t)=-3 \eta(t)-e^{-t^{2}} \eta(t)+\frac{1}{6} e^{-t^{2}} \zeta(t-1)-\frac{1}{8} \dot{\eta}(t-1) .
\end{aligned}
$$

Clearly, the given system can be rewritten as

$$
\frac{d}{d t}(x(t)-D x(t-1))=A(t) x(t)+B(t) x(t-1)+\int_{-1}^{0} C(t) x(t+s) d s,
$$


where $x(t):=(\zeta(t), \eta(t))^{T} \in \mathbb{R}^{2}, t \in \mathbb{R}$ and

$$
\begin{gathered}
D:=\left(\begin{array}{cc}
\frac{1}{4} & 0 \\
0 & -\frac{1}{8}
\end{array}\right) ; \quad A(t):=\left(\begin{array}{cc}
-4-\sin t & 0 \\
0 & -3-e^{-t^{2}}
\end{array}\right), \\
B(t):=\left(\begin{array}{cc}
0 & -\frac{1}{t^{2}+16} \\
\frac{1}{6} e^{-t^{2}} & 0
\end{array}\right) ; \quad C(t):=\left(\begin{array}{cc}
0 & \frac{1}{16} e^{-t^{2}} \\
0 & 0
\end{array}\right) .
\end{gathered}
$$

Let us define

$$
A:=\left(\begin{array}{cc}
-3 & 0 \\
0 & -3
\end{array}\right) ; \quad B:=\left(\begin{array}{cc}
\frac{5}{4} & \frac{1}{8} \\
\frac{1}{6} & \frac{1}{2}
\end{array}\right) \text {. }
$$

It is easy to check that $\rho(|D|)<1$ and

$$
M(A(t)) \leq A, \quad \forall t \in \mathbb{R} ; \quad|A(t) D|+|B(t)|+\int_{-1}^{0}|C(t)| d s, \leq B, \quad \forall t \in \mathbb{R} .
$$

Thus, 30 holds. By simple computation, we have

$$
A+B\left(I_{2}-|D|\right)^{-1}=\left(\begin{array}{cc}
-\frac{4}{3} & \frac{1}{7} \\
\frac{2}{9} & -\frac{17}{7}
\end{array}\right) .
$$

Because of

$$
\left(\begin{array}{cc}
-\frac{4}{3} & \frac{1}{7} \\
\frac{2}{9} & -\frac{17}{7}
\end{array}\right)\left(\begin{array}{l}
1 \\
1
\end{array}\right) \ll\left(\begin{array}{l}
0 \\
0
\end{array}\right),
$$

the Metzler matrix $A+B\left(I_{2}-|D|\right)^{-1}$ is Hurwitz stable, by Theorem 2.1. Thus, the given system is exponentially stable, by Theorem 3.8 (ii).

Finally, a remarkable result on asymptotic stability of the linear neutral time-invariant differential systems can be found in [17]. It has been shown [17, Theorem 3.1] that (33) with $C(\cdot) \equiv 0$ is asymptotically stable if

$$
\|D\|<1 \quad \text { and } \quad \mu(A)+\frac{\|B\|+\|A\|\|D\|}{1-\|D\|}<0,
$$

where $\mu(A):=\lim _{\epsilon \rightarrow 0^{+}} \frac{\left\|I_{n}+\epsilon A\right\|-1}{\epsilon}$ is the matrix measure of $A$. It is quite easy to show that (42) is more conservative than (34) (with $C(\cdot) \equiv 0$ ).

\section{CONCLUding REMARK}

In this paper, we have addressed the challenging problem of stability analysis for linear non-autonomous systems described by functional differential equations of neutral type and presented some novel conditions for exponential stability of time-varying functional differential equations. By using a system transformation, the comparison principle and spectral properties of Metzler matrices, the resulting criteria are both generic and explicitly obtained. As such, the proposed approach can be extended to study problems of exponential stability of nonlinear and singular time-varying systems, which will be subject to our future work. 


\section{REFERENCES}

[1] R.P Agarwal, S.R Grace, Asymptotic stability of certain neutral differential equations, Mathematical and Computer Modelling 31 (2000), 9-15.

[2] A. Bellen, N. Guglielmi and A.E. Ruehli, Methods for linear systems of circuit delay differential equations of neutral type, IEEE Transactions on Circuits and Systems: Fundamental theory and Applications, 46 (1999), 212-216.

[3] A. Berman, R.J. Plemmons, Nonnegative Matrices in Mathematical Sciences, Acad. Press, New York, 1979.

[4] S. Cong, On exponential stability conditions of linear neutral stochastic differential systems with time-varying delay, International Journal of Robust and Nonlinear Control 23 (2013), 1265-1276.

[5] M.A. Cruz, J.K. Hale, Stability of functional differential equations of neutral type, Journal of Differential Equations 7 (1970), 334-355.

[6] J. Duda, A Lyapunov functional for a neutral system with a time-varying delay, Bulletin of the Polish Academy of Sciences, Technical Sciences 61 (2013), 911-918.

[7] E. Fridman, New Lyapunov-Krasovskii functionals for stability of linear retarded and neutral type systems, Systems Control Letters 43 (2001), 309-319.

[8] M.I. Gil, Stability of Neutral Functional Differential Equations, Atlantis Press, 2014.

[9] J.K. Hale and S.M.V. Lunel, Introduction to Functional Differential Equations, Springer-Verlag Berlin, Heidelberg, New york, 1993.

[10] Q.L. Han, Improved stability criteria and controller design for linear neutral systems, Automatica 45 (2009), 1948-1952.

[11] E. Hewitt, K. R. Stromberg, Real and Abstract Analysis, Springer-Verlag, New York, 1965.

[12] D. Ivanescu, S.I. Niculescu, Luc Dugard, J. M. Dion, E.I. Verriest, On delay-dependent stability for linear neutral systems, Automatica 39 (2003), 255-261.

[13] A.F. Ize, J.G. Dos Reis, Contributions to stability of neutral functional differential equations, Journal of Differential Equations 29 (1978), 58-65.

[14] V.B. Kolmanovskii and V. R. Nosov, Stability of Functional Differential Equations, Academic Press 1986.

[15] V.B. Kolmanovskii and A. Myshkis, Applied Theory of Functional Differential Equations, Dordrecht, the Neitherlands: Kluwer 1996.

[16] V. Kharitonov, S. Mondie, J. Collado, Exponential estimates for neutral time-delay systems: An LMI Approach, IEEE Transactions on Automatic Control 50 (2005), 666-670.

[17] L.M. Li, Stability of linear neutral delay-differential systems, Bulletin of the Australian Mathematical Society 38 (1988), 339-344.

[18] X.G. Liu, M. Wu, R. Martin, M.L. Tang, Stability analysis for neutral systems with mixed delays, Journal of Computational and Applied Mathematics 202 (2007), 478-497,

[19] S.I. Niculescu, B. Brogliato, Force measurement time-delays and contact instability phenomenon, European Journal of Control 5 (1999), 279-289.

[20] S.A. Rodrguez, J.M. Dion, L. Dugard, Stability of Neutral Time Delay Systems: A Survey of Some Results, Advances in Automatic Control 754 (2003), 315-335. 